\title{
Le corps dégénéré : des textes scientifiques à la littérature française du XIX ${ }^{e}$ siècle
}

\section{Bénédicte Percheron}

\section{(2) OpenEdition \\ Journals}

Édition électronique

URL : http://journals.openedition.org/aes/935

DOI : $10.4000 /$ aes.935

ISSN : 2258-093X

Éditeur

Laboratoire LISAA

Référence électronique

Bénédicte Percheron, "Le corps dégénéré : des textes scientifiques à la littérature française du xIx ${ }^{\mathrm{e}}$ siècle ", Arts et Savoirs [En ligne], 7| 2016, mis en ligne le 13 décembre 2016, consulté le 10 décembre 2020. URL : http://journals.openedition.org/aes/935; DOI : https://doi.org/10.4000/aes.935

Ce document a été généré automatiquement le 10 décembre 2020.

Centre de recherche LISAA (Littératures SAvoirs et Arts) 


\title{
Le corps dégénéré : des textes scientifiques à la littérature française du XIX ${ }^{\mathrm{e}}$ siècle
}

\author{
Bénédicte Percheron
}

1 Au début du XIX ${ }^{e}$ siècle, avec la poussée des théories transformistes, naturalistes, écrivains et philosophes s'interrogent sur le devenir de l'homme. La question primordiale est de savoir si l'homme est physiquement achevé, comme l'affirment les textes bibliques. Ce sont les premières discussions sur les variations du vivant qui provoquent, parfois avec quelques années de décalage, ces débats autour d'un sujet condamné par l'Église. C'est en effet un petit paragraphe du tome XIV de L'Histoire naturelle de Buffon, paru en 1766, qui émet l'idée d'une dégénération pour expliquer la variété du vivant. Le topos de la dégénération, appelée plus tard dégénérescence, si apprécié des écrivains $\mathrm{du} \mathrm{XIX}^{\mathrm{e}}$ siècle, se forme bien dans l'histoire naturelle au cœur même des idées pré-transformistes du siècle précédent. Si la notion est tout d'abord appliquée à l'espèce animale, elle est utilisée ensuite dans le domaine médical, ce qui permet finalement un glissement dans le domaine de la psychologie et de la morale. L'idée n'est plus seulement appliquée à un groupe, mais à un individu humain. À la suite du texte de Buffon, les expressions « dégénération » et " dégénérescence » se multiplient dans les textes scientifiques, mais aussi littéraires. Certains contestent toutefois l'idée unique d'une dégénération pour expliquer la diversité du vivant, car une modification n'est pas toujours négative. L'homme pourrait aussi se perfectionner, aussi bien sur le plan physique, qu'intellectuel. Ces notions de dégénération et de perfection, utilisées par les écrivains, soulèvent de nombreuses questions tant ces conceptions se prêtent à des usages et des interprétations parfois très différents. Tantôt récupérés par des pensées religieuses et conservatrices, aussi bien en philosophie qu'en littérature, les concepts de dégénération et, par opposition, de perfectibilité1, prouvent que le vivant et sa temporalité propre sont au cœur des débats du XIX ${ }^{e}$ siècle. Quelles sont les images littéraires qui ont été suscitées par ces notions? Comment les écrivains les ont-ils utilisées pour imaginer l'évolution du corps humain et pour rêver l'avenir de l'Homme? Quelques exemples nous permettront 
d'observer les transferts de savoirs biologiques et médicaux vers les textes littéraires français du XIX ${ }^{e}$ siècle ${ }^{2}$. Tout d'abord, il s'agira de voir comment la notion de dégénération a pu, à une certaine époque, expliquer l'existence de variations au sein des espèces, aussi bien dans les écrits des naturalistes que dans ceux des écrivains. Les premières utilisations de la notion de dégénération, dans le Génie du Christianisme de Chateaubriand ${ }^{3}$ par exemple, qui cherchent souvent à expliquer les imperfections de la société, cèdent la place à des fictions, qui sont parfois des romans d'anticipation, surtout à partir des années 1830. Ces spéculations s'appuieront sur les théories biologiques de la dégénération. Enfin, il nous faudra nous intéresser aux représentations dans la littérature française de la dégénérescence appliquée au champ médical.

\section{Buffon : principe de variation par la dégénération}

En 1766, Buffon tente de fournir une explication des variations dans l'espèce humaine par un phénomène qu'il nomme " dégénération » :

De la dégénération des Animaux.

Dès que l'Homme a commencé à changer de ciel, et qu'il s'est répandu de climats en climats, sa nature a subi des altérations : elles ont été légères dans les contrées tempérées, que nous supposons voisines du lieu de son origine: mais elles ont augmenté à mesure qu'il s'en est éloigné ; et lorsqu'après des siècles écoulés, des continens traversés et des générations déjà dégénérées par l'influence des différentes terres, il a voulu s'habituer dans les climats extrêmes, et peupler les sables du Midi et les glaces du Nord; les changemens sont devenus si grands et si sensibles, qu'il y auroit lieu de croire que le Nègre, le Lappon et le Blanc forment des espèces différentes, si d'un côté l'on n'étoit assuré qu'il n'y a eu qu'un seul Homme de créé, et de l'autre que ce Blanc, ce Lappon et ce Nègre, si dissemblans entr'eux, peuvent cependant s'unir ensemble et propager en commun la grande et unique famille de notre genre humain : ainsi leurs taches ne sont point originelles; leurs dissemblances n'étant qu'extérieures, ces altérations de nature ne sont que superficielles; et il est certain que tous ne font que le même homme, qui s'est verni de noir sous la zone Torride, et qui s'est tanné, rapetissé par le froid glacial de la sphère du Pôle. ${ }^{4}$

La reconnaissance d'altérations corporelles induites par le climat, l'alimentation ou encore la domestication paraît remettre en cause l'idée d'une fixité de l'espèce, mais Buffon reste prudent en évoquant plutôt, tout le long de l'édition de son Histoire naturelle, un monde en déclin, en raison de la disparition des espèces de grande taille ${ }^{5}$. Il donne pour argument la diminution de la taille de l'éléphant et de l'hippopotame par rapport à ce qu'il nomme leurs « dépouilles antiques $»^{6}$.

En France, la notion est mise de côté pendant plusieurs décennies, mais en Allemagne, un des pionniers de l'anthropologie, Johann Blumenbach, reprend la théorie de Buffon pour expliquer les variations physiques des êtres humains dans sa thèse de médecine de $1775^{7}$. Il utilise ainsi le principe de dégénération pour expliquer les variations au sein de l'espèce humaine, qu'il divise en cinq variétés (figure 1). Il y a selon lui une unité de l'espèce humaine, mais les différentes conditions de vie, notamment l'air atmosphérique, ont fait varier les humains 8 . Selon Blumenbach, la dégénération a lieu quand il y a éloignement de la variété caucasienne, considérée implicitement comme la race d'origine. Son modèle primitif de référence demeure, pour lui, la race géorgienne. La traduction de sa thèse en France en 1804 semble avoir relancé le débat et inspiré de nouveaux écrits. 


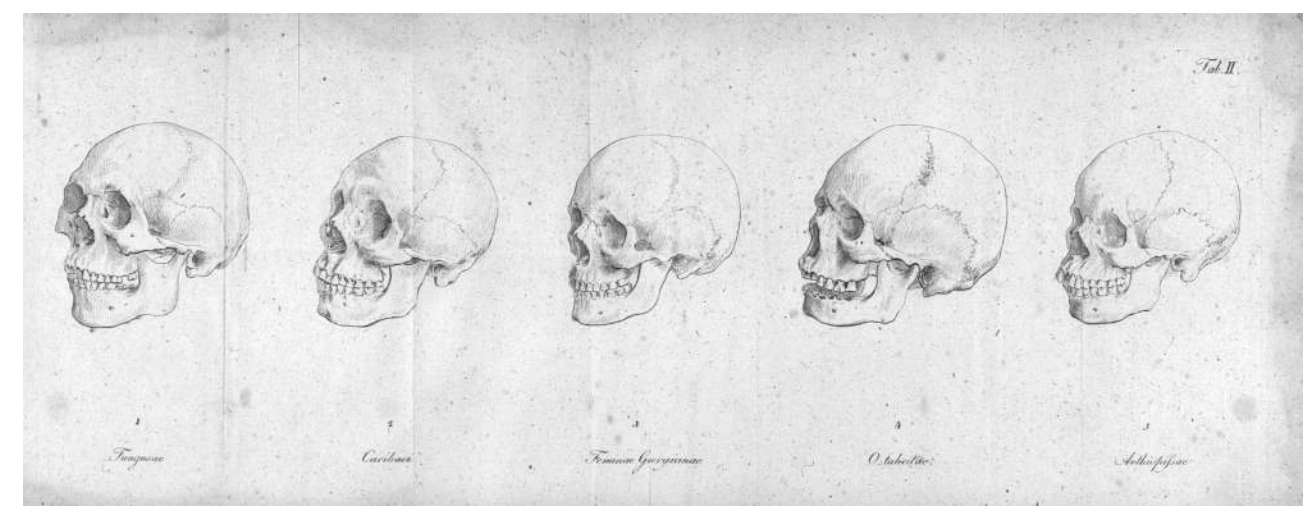

Figure 1

Johann Blumenbach, De generis humani varietate nativa, Göttingen, Vandenhoek et Ruprecht, 1775, 398 p.

Collection BIU Santé Médecine, licence ouverte

6 Dès 1806, Jean-Claude de La Métherie ${ }^{9}$ consacre un ouvrage entièrement dédié au phénomène. Intitulé Considérations sur les êtres organisés: de la perfectibilité et de la dégénérescence des êtres organisés ${ }^{10}$, il délaisse le terme de dégénération au profit de dégénérescence, qui semble prendre le dessus sur le premier mot au milieu des années $1830^{11}$. L'occurrence « dégénérescence » domine amplement dans les textes français dans les années 1860, après les écrits de l'aliéniste Bénédict-Auguste Morel. Dès lors, les notions de dégénération et de dégénérescence deviennent particulièrement ambiguës. Dans le Nouveau dictionnaire d'histoire naturelle de Julien-Joseph Virey de 1817, le terme dégénération est un synonyme de variation ${ }^{12}$. Virey insiste sur le caractère monstrueux de la dégénération, car l'individu, trop écarté de son type primitif est de surcroît stérile. Le responsable de cet état est «la domesticité [qui] dégrade et vicie "13. L'animal remis dans la nature subit une régénération, en retrouvant ses caractéristiques primitives.

7 Mais en 1844, dans le Dictionnaire universel d'histoire naturelle ${ }^{14}$ publié sous la direction de Charles d'Orbigny, le botaniste Frédéric Gérard, se détache des définitions délivrées par ses contemporains. Gérard s'oppose à l'idée même de dégénérescence qui n'engendre que des dégradations, car selon lui, les modifications organiques peuvent aussi être «un passage à une condition meilleure $»^{15}$. Il estime même que le terme " commence à tomber en désuétude $\aleph^{16}$, surtout après les écrits transformistes de Jean-Baptiste de Lamarck et d'Étienne Geoffroy Saint-Hilaire. Il va plus loin en affirmant que les notions d'anomalies, de monstruosités, de dégénération sont des erreurs dues aux notions de fixité de l'espèce et d'harmonie de la nature qu'il rejette.

\section{Premiers transferts : dégénération et déclin}

8 À la fin du XVIII ${ }^{\mathrm{e}}$ siècle, la dégénération repose avant tout sur les textes bibliques et fait écho à l'idée de la chute de l'Homme qui suit le péché originel. Après leur éviction du paradis, Adam et Ève, ainsi que leurs descendants, ont perdu leur perfection originelle. En effet, si l'homme est fait à l'image de Dieu, la dégénération explique les défauts humains. C'est ainsi que François-René de Chateaubriand entame, en 1802, le troisième chapitre du Génie du christianisme intitulé «Constitution primitive de l'homme. Nouvelle preuve du 
péché originel ». L'auteur oppose l'ordre de l'univers, l'harmonie de la nature, aux défauts humains, qui sont « une preuve nouvelle de notre dégénération primitive $»^{17}$.

L'homme tel que nous le voyons n'est vraisemblablement pas l'homme primitif. Il contredit la nature: déréglé quand tout est réglé, double quand tout est simple, mystérieux, changeant, inexplicable, il est visiblement dans l'état d'une chose qu'un accident a bouleversée : c'est un palais écroulé et rebâti avec ses ruines [...].18

Cette idée réapparaît au XIX ${ }^{\mathrm{e}}$ siècle chez plusieurs auteurs. Nodier, fervent croyant et avide lecteur d'ouvrages naturalistes ${ }^{19}$, remploie la notion dans la Fée aux Miettes, en 1832 :

Il faut convenir, ajoutai-je à demie-voix, sans abandonner cette pensée, que le mystère du sixième jour de La Genèse est encore loin d'être éclairci, et qu'en réduisant l'homme dégradé par sa faute à l'état des animaux relevés jusqu'à son abaissement, le Seigneur aurait tiré une digne vengeance de l'orgueil insensé du père de notre race. - Et alors, ou je me trompe, les enfants d'Adam qui auraient conservé sans altération, pendant la nouvelle épreuve de la vie, le germe de l'immortalité qui a été déposé en eux, pourraient espérer de retourner un jour à ce paradis de délices, œuvre facile de la toute-puissance, œuvre nouvelle de la toutebonté. Le reste retournerait d'où il vient : dans le foyer de la matière éternelle !20

Nodier va donc plus loin et imagine qu'il existe peut-être des hommes non dégénérés sur terre qui pourraient regagner le paradis terrestre.

Chez Chateaubriand, la dégénération est cependant plus morale que physique. Tout comme Buffon, il parle d'un « monde dégénéré $~^{21}$, néanmoins pas pour évoquer une sorte d'essoufflement de la nature qui se manifeste par une diminution physique, mais pour présenter une civilisation en déliquescence morale. Le remède à ce mal serait un renouvellement du christianisme, par une nouvelle prédication de l'Évangile ${ }^{22}$. Cette application de l'idée de dégénération aux civilisations s'accentue dans les années 1810, sous l'impulsion d'écrivains conservateurs. La figure de Louis de Bonald, homme politique contre-révolutionnaire et écrivain, illustre cette mouvance. Ses essais n'ont de cesse de critiquer l'idéologie révolutionnaire, à l'origine de la destruction d'une unité nationale, au profit d'un épanouissement personnel qu'il juge égoïste. Il milite de fait pour la conservation de codes moraux, dont la monarchie ou encore l'Église étaient garantes. Il est par ailleurs l'un des plus fervents diffuseurs du concept de déclin national du début du $\mathrm{XIX}^{\mathrm{e}}$ siècle ${ }^{23}$. En 1810, à travers un essai économique et social intitulé « Du perfectionnement de l'homme ", il défend son idée de dégénération de la société, malgré une multiplication des progrès techniques qu'il ne nie pas, en arguant qu'une amélioration matérielle peut vider le contenu de ses qualités artistiques ou morales :

Je dis plus : toutes les sociétés glissent sur une pente rapide sur laquelle il leur est impossible de s'arrêter, et elles seront de chute en chute entraînées au bien ou au mieux par des révolutions, si elles ne s'y laissent pas conduire par la raison.

Les efforts que tous les gouvernements font depuis soixante ans, à l'envi les uns des autres, pour améliorer l'état de la société, ont eu, il en faut convenir, de plus heureux effets sur le matériel de la société que sur le moral; les arts aujourd'hui comptent plus de chefs-d'œuvre que les lettres; on imprime mieux qu'on ne compose ; il y a dans nos ballets plus de génie et d'invention que dans nos tragédies. 24

12 S'il reconnaît les améliorations matérielles de l'ensemble de la société, il considère que le matériel n'a favorisé finalement que les arts mineurs au détriment de ceux utiles à la morale.

13 Cette idée de déclin s'est de même transmise aux discours, scientifiques ou fictionnels, appliqués à des peuples extra-européens. L'exemple le plus remarquable du XIX ${ }^{e}$ siècle 
dérive d'un fait réel, largement diffusé par la presse et par la photographie (figure 2). Il s'agit de la découverte et de l'exhibition publique des deux microcéphales des salvadoriens Maximo et Bartola, exhibés aux États-Unis à partir de la fin de l'année 1848, puis présentés sur les champs de foire européens en 1853. Les deux êtres sont alors désignés comme les derniers aztèques existants et sont ainsi la preuve d'une dégénérescence de leur civilisation ${ }^{25}$. Bien que la supercherie ait été dès le début mise au jour par les scientifiques ${ }^{26}$, les exhibitions de ces personnalités connaissent un important succès jusqu'au début du $\mathrm{Xx}^{\mathrm{e}}$ siècle, période où ces phénomènes disparaissent des foires ${ }^{27}$. L'histoire a, entre autres, donné naissance à des récits plus ou moins réels de leur découverte. En 1855, un écrivain et journaliste français, Bénédict Révoil ${ }^{28}$, s'empare de cette histoire pour la livrer au lectorat français. En 1849, Révoil avait en effet assisté à plusieurs reprises à la présentation des Salvadoriens. Il propose ainsi dans la préface et la postface de son ouvrage Les Aztecs ${ }^{29}$ une histoire de la découverte de ces deux personnages beaucoup plus crédible que celle avancée par Morris, leur impresario.

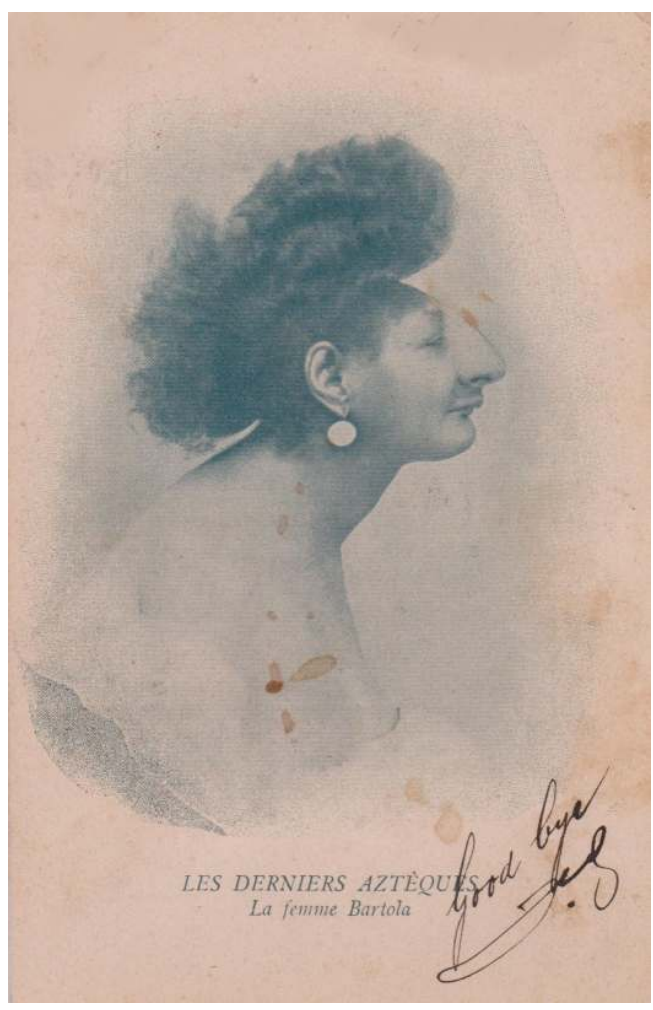

Figure 2

Bartola, la femme aztèque, carte postale, c. 1900

Collection de l'auteur

Enfin, sur les pas de Blumenbach, les théoriciens des races humaines mobilisent encore ce concept pour hiérarchiser les êtres humains. En 1853, dans son Essai sur l'inégalité des races humaines, Arthur de Gobineau affirme encore :

Je pense donc que le mot dégénéré, s'appliquant à un peuple, doit signifier et signifie que ce peuple n'a plus la valeur intrinsèque qu'autrefois il possédait, parce qu'il n'a plus dans ses veines le même sang, dont des alliages successifs ont graduellement modifié la valeur ; autrement dit, qu'avec le même nom, il n'a pas conservé la même race que ses fondateurs; enfin, que l'homme de la décadence, celui qu'on appelle 
l'homme dégénéré, est un produit différent, au point de vue ethnique, du héros des grandes époques $[\ldots] .^{30}$

Pour Gobineau, ce sont ainsi les métissages avec d'autres ethnies qui auraient provoqué la dégénération et donc la chute de nombreux peuples.

\section{Perfectibilité contre dégénération : les possibilités de l'évolution humaine}

16 Dans les années 1830, la question de la dégénération devient ardente suite aux débats liés au transformisme de Lamarck, relancés par celui de l'unité de plan d'Étienne Geoffroy Saint-Hilaire. Plusieurs écrivains se passionnent pour l'idée de changement corporel et imaginent les possibilités évolutives de l'Homme. Pour certains, le corps humain peut connaître soit une dégradation physique ou, a contrario, un perfectionnement lui permettant d'augmenter ses capacités physiques ou intellectuelles.

Les visions les plus pessimistes envisagent l'extinction de l'homme, de la même façon dont des animaux ont disparu par le passé, comme le témoignent les fossiles. Dans De Palingénésie humaine et de la Résurrection de Nodier (1832), l'homme peut soit disparaître, soit descendre dans l'échelle des êtres au niveau des organismes les plus simples, comme les polypes ou encore les monades, ce qui formerait une nouvelle espèce :

Non! Il n'y a point de palingénésie spécifique pour l'organisation actuelle de l'homme, parce que l'homme approche du temps où il aura fini son rôle sur la terre, comme le reste des animaux fantastiques du monde fossile, à moins qu'il ne redescende, brut et sauvage, à la tête des espèces inférieures, pour faire place à une espèce nouvelle. $^{31}$

Si certains voient un rabaissement de l'homme au rang des animaux, d'autres, surtout après les écrits de Darwin, affirment, au contraire, comme Claparède, qu'il « vaut mieux être un singe perfectionné qu'un Adam dégénéré $\|^{32}$. L'homme tombé de son piédestal demeure toutefois une espèce à part au XIX ${ }^{\mathrm{e}}$ siècle. Aux différentes interprétations du darwinisme s'ajoute le syncrétisme volontaire de certains écrivains. Ainsi, Maupassant, par exemple, mêle l'évolution à la métempsychose dans Le docteur Héraclius Gloss, et le sens de la dégénération est inversé. L'homme est cette fois-ci dégénéré par rapport à l'animal :

Il prétendait que l'abbé Rosencroix, le premier vicaire de Sainte-Eulalie, avait été indubitablement une corneille qui abat des noix, car il en avait conservé la robe et les attributions. Puis, intervertissant les rôles de la façon la plus déplorable, il affirmait que maître Bocaille, le pharmacien, n'était qu'un ibis dégénéré, puisqu'il était contraint de se servir d'un instrument pour infiltrer ce remède si simple que, suivant Hérodote, l'oiseau sacré s'administrait lui-même avec l'unique secours de son bec allongé. ${ }^{33}$

Mais certains écrivains donnent à la notion de dégradation une acception plus sociale, comme Hugo. Dans l'exergue des Misérables de 1862, il évoque les trois problèmes du siècle : « la dégradation de l'homme par le prolétariat, la déchéance de la femme par la faim, l'atrophie de l'enfant par la nuit ${ }^{34}$. Ce sont les conditions difficiles de vie des ouvriers qui provoquent leur dégradation physique. Cette remarque fait écho aux recherches de nombreux médecins du milieu du XIX ${ }^{\mathrm{e}}$ siècle sur l'influence du travail sur la santé ouvrière, notamment à celles menées par Morel dans son Traité des dégénérescences physiques, intellectuelles et morales et des causes qui produisent ces variétés maladives ${ }^{35}$. En effet, en 1857, ce médecin reprend le concept de Buffon et l'étend au domaine de la médecine. 
Ce traité connaît un retentissement important dans la société, notamment chez les écrivains : Zola le lit et utilise ses idées dans plusieurs de ses ouvrages comme nous le verrons ultérieurement.

L'espoir d'une perfectibilité de l'espèce humaine s'oppose à la dégénération. Au-delà des perfections sociétales, plusieurs écrivains rêvent d'un nouveau corps amélioré, influencé parfois par l'anatomie d'autres espèces animales. On peut le percevoir chez Nodier. Dans son essai philosophique Palingénésie, il discute de ses possibilités évolutives. Il imagine ainsi un homme qu'il qualifie de compréhensif. Il s'agit d'un état « intermédiaire entre l'état pensant et l'état résurrectionnel ${ }^{36}$, qui aura la capacité de se comprendre luimême. Des modifications corporelles le libéreront de ses contraintes physiques actuelles. Ce nouvel homme aura aussi tous les sens des êtres créés, ainsi que d'autres encore que nous ignorons. Son appareil respiratoire sera aussi « une fonction facultative $»^{37}$ ce qui lui permettrait de vivre sous l'eau. À la place de ses poumons, un large espace, que l'homme pourrait vider à son gré, lui permettra de s'envoler dans les airs. Mais Nodier, conservateur, semble surtout forcer le trait pour ridiculiser le transformisme.

21 Vingt ans après Nodier, l'homme compréhensif devient sous la plume de Louis Bouilhet l'« être universel $\aleph^{38}$. Dans son poème Les fossiles, parus en 1854, Bouilhet décrit des caractéristiques physiques de cet " être nouveau », qui « a l'instinct sublime et la sagesse immense ». Libéré des contraintes des lois de la physique :

Il bondit sur les monts, tel qu'un chamois rapide,

Il nage dans l'azur, aux grands aigles mêlé,

Il marche au fond du fleuve, et sa forme splendide

Luit à travers les flots comme un ciel étoilé. ${ }^{39}$

À partir des années 1880, on imagine des hommes capables de télépathie. Le journaliste et écrivain Louis Boussenard met en scène cette hypothèse dans 10000 ans dans un bloc de glace. Comme le titre le suggère, un savant endormi dans la glace depuis 10000 ans se réveille et découvre les hommes du futur. Dotés d'une intelligence supérieure, ils volent, se déplacent aussi vite que la pensée et sont télépathes. Physiquement, ils sont dotés de têtes aux dimensions « énormes ${ }^{40}$. Ce sont des métis africano-asiatiques, car l'Europe a disparu recouverte sous la glace. Quant aux Européens survivants, devenus esclaves, ils ont probablement "dégénéré" au profit des populations des autres continents ${ }^{41}$. La lecture des œuvres de Louis Boussenard est très intéressante du point de vue de la réception des savoirs scientifiques et de leur diffusion au grand public, car il fait constamment la synthèse de très nombreuses théories scientifiques contemporaines et les met en fiction. Il faut noter que cette œuvre est probablement inspirée d'une série de contes satiriques de Nodier, intitulée Hurlubleu et autres contes du dériseur sensét $e^{42}$, dont les premières nouvelles paraissent en 1833 dans la Revue de Paris. Nodier y tourne en ridicule les visions progressistes de l'Homme et de façon plus générale l'hypothèse de la perfectibilité.

\section{Déplacement de la notion : la dégénérescence médicale}

En 1857, B.-A. Morel reprend la notion utilisée par Buffon et l'étend au champ médical. Selon lui, la dégénérescence serait due, entre autres, aux conditions de logement, au lieu de travail et à l'alcoolisme ${ }^{43}$. Par de nombreux exemples issus d'observations zoologiques, ainsi qu'en utilisant des études ethnographiques, il cherche à montrer que les habitudes 
acquises par l'éducation se transmettent de génération en génération. C'est pourquoi il souhaite appliquer un traitement moral pour soigner les maladies mentales et surtout cette dégénérescence qui s'accentue avec les générations. Il développe des propos préeugénistes, mais il précise qu'il faut se préserver de certaines pratiques pour pallier l'abâtardissement de l'espèce humaine. En effet, selon lui, «l'existence des êtres dégénérés est nécessairement bornée, et, chose merveilleuse, il n'est pas toujours nécessaire qu'ils arrivent au dernier degré de la dégradation pour qu'ils restent frappés de stérilité, et conséquemment incapables de transmettre le type de leur dégénérescence $~^{44}$. Cette fois-ci la dégradation n'est pas une dégradation dans l'échelle des classifications zoologiques de cette époque, mais bien un synonyme de dégénérescence. Par la suite, Charles Féré, combine la dégénérescence selon Morel à l'évolution darwinienne, ce qui engendre une forme d'évolution régressive. L'homme dégénéré a alors des comportements courants chez les singes, comme l'apathie. Inadapté à la société, il ne peut que naturellement disparaitre ${ }^{45}$. Cette inadaptation aux exigences de la société est flagrante dans L'Assommoir de Zola. Coupeau, après la chute du toit, prend goût à ne rien faire et s'adonne à la boisson. Sous les effets de l'alcool, son visage gagne en caractéristique simiesque, si bien qu'il a désormais une «mâchoire de singe $»^{46}$. Gervaise, après la mort de son mari, adopte les mêmes travers et sombre d'une façon similaire :

Ça se bornait à des grimaces de singe échappé, qui lui faisaient jeter des trognons de choux par les gamins, dans les rues. Gervaise dura ainsi pendant des mois. Elle dégringolait plus bas encore, acceptait les dernières avanies, mourait un peu de faim tous les jours. ${ }^{47}$

24 La chute du toit engendre en quelque sorte une chute dans l'échelle des êtres; une dégradation au niveau des animaux considérés comme inférieurs.

\section{Hérédité et roman moral}

Le concept d'hérédité morbide connaît un retentissement certain dans la société occidentale de la fin du xix siècle et, contrairement au transformisme, il est rarement contesté. Flaubert le convoque dans Bouvard et Pécuchet pour expliquer l'échec de l'éducation de Victor et Victorine. Après que Victor a mis le chat dans la marmite, et donc provoqué la mort de l'animal d'une façon atroce et sadique, Bouvard et Pécuchet cherchent à comprendre ce comportement. Ils concluent que désormais « le sang paternel se manifestait $»^{48}$. Tous les comportements du garçon ramènent par ailleurs au statut de forçat du père. Quant à Victorine, elle est corrompue et vicieuse. À propos des deux enfants, Pécuchet finit par dire: «Mais il y a des natures dénuées de sens moral; - et l'éducation n'y peut rien $»^{49}$.

Si Flaubert use avec parcimonie de la théorie de la dégénérescence, Zola l'inclut dans un système de création littéraire beaucoup plus vaste ${ }^{50}$, au cœur même d'un cycle d'ouvrages en vingt volumes, celui des Rougon-Macquart. Plusieurs personnages y sont particulièrement frappés d'hérédité morbide. Dans Au bonheur des Dames, de 1883, la description de Madame Bandu et de sa fille insiste sur l'accentuation des tares familiales d'une génération à une autre :

La première était une petite femme mangée d'anémie, toute blanche, les cheveux blancs, les lèvres blanches. Geneviève, chez qui s'aggravait encore la dégénérescence de sa mère, avait la débilité et la décoloration d'une plante grandie à l'ombre. ${ }^{51}$ 
le mal suprême qui corrompt le plus les lignées généalogiques demeure l'alcoolisme. Germinal s'attache à montrer les conséquences de l'alcool sur les enfants de Gervaise, précisément sur Étienne, qui explique à Catherine l'état de folie incontrôlé et fatal dans lequel la boisson le plonge :

Je dois dire que j'avais bu, continua-t-il, et quand je bois, cela me rend fou, je me mangerais et je mangerais les autres... oui, je ne peux pas avaler deux petits verres, sans avoir le besoin de manger un homme... ensuite, je suis malade pendant deux jours. - Il ne faut pas boire, dit-elle sérieusement. - Ah! N'aie pas peur, je me connais ! Et il hochait la tête, il hochait la tête, il avait une haine de l'eau-de-vie, la haine du dernier enfant d'une race d'ivrognes, qui souffrait dans sa chair de toute cette ascendance trempée et détraquée d'alcool, au point que la moindre goutte en était devenue pour lui un poison. ${ }^{52}$

Enfin, Zola arrive au bout de son étude d'histoire naturelle des familles RougonMarcquart dans le Docteur Pascal, où le médecin de la famille procède à un inventaire de toutes les tares familiales ${ }^{53}$. Le souhait du docteur est de trouver un remède à la dégénérescence. Sa nièce, Clotilde, demeure son sujet d'expérimentation le plus abouti. Pour éviter la reproduction des tares de ces parents, il a soustrait la jeune femme à sa famille, ce qui conduit finalement à une autre forme de honte sociale: une relation incestueuse avec sa propre nièce.

Si Zola est ambigu sur la portée morale de cette situation, les termes de dégénérescence, dégénération ou dégradation sont, de façon générale dans la littérature, souvent adjoints au champ lexical de la débauche. La syphilis est par excellence la marque physique, visible, d'un comportement moral réprouvé par la société. Mais la maladie résultant de cette dégénération morale qui se manifeste par des stigmates physiques peut parfois donner lieu, de façon étonnante, à de la poésie. Ainsi, sur le modèle du poème fondateur de cette étrange thématique, Syphilis de l'Italien Fracastor de $1530^{54}$, le médecin Auguste Barthélemy propose un poème en quatre chants, au cours duquel il affirme : "Jamais la dégénération de l'espèce humaine ne s'était montrée sous des formes aussi hideuses que de nos jours $»^{55}$. La poésie devient alors une œuvre médicale et morale. 


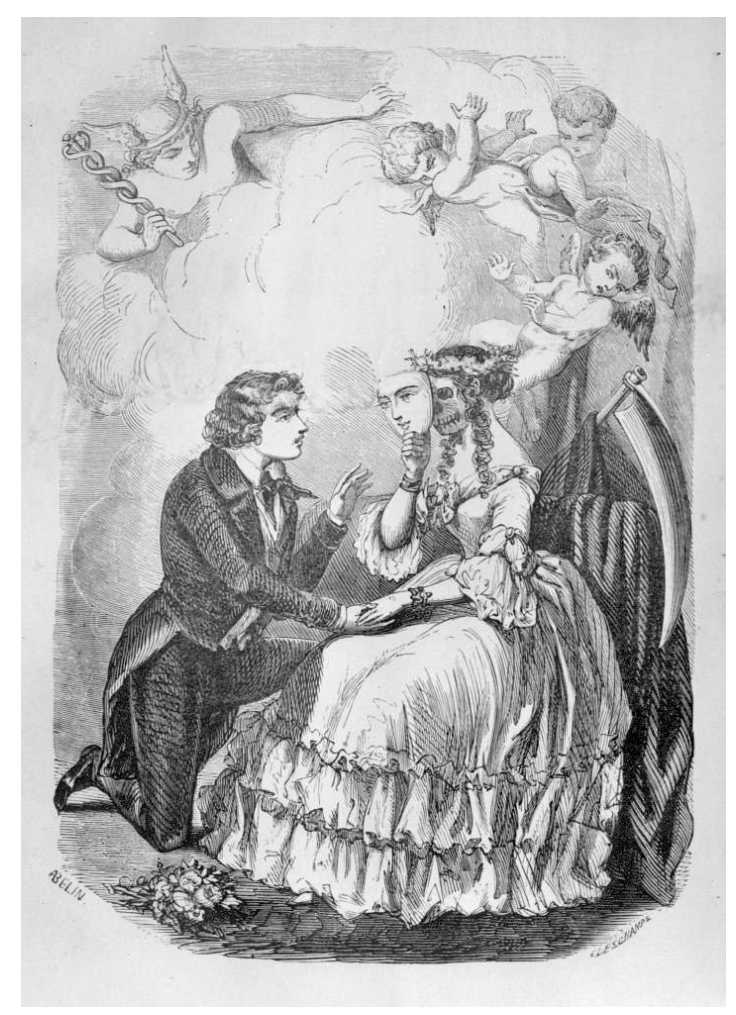

Figure 3

Dessin d'A. Belin, frontispice d'Auguste, Barthélemy, Syphilis : poème en deux chants, Paris, Martinon, 1851

Collection BIU Santé Médecine, licence ouverte

\section{Le dégénéré décadent selon Nordau}

Avec la littérature fin de siècle, le personnage de Des Esseintes créé par Huysmans dans À rebours devient, selon Max Nordau, la figure dégénérée, décadente par excellente. Si Huysmans présente son personnage comme un aristocrate dégénéré, Nordau montre surtout que c'est l'attitude du protagoniste, centré sur lui-même, qui est un signe de décadence, car l'individu se désintéresse de l'avenir de ses semblables. Ce n'est ni Darwin, ni Spencer ${ }^{56}$ que Nordau cite, mais Paul Bourget, théoricien de la décadence, et son Essai de psychologie contemporaine dans lequel il affirme qu'une « société doit être assimilée à un organisme ${ }^{57}$. Le dégénéré décadent est donc inutile à la société : cette figure lui est même nocive car elle la pervertit, en corrompant ses repères moraux. Nordau qualifie des Esseintes de «[p]arasite de la plus basse espèce, sorte de sacculus humain, il serait condamné, s'il était pauvre, à mourir misérablement de faim, au cas où la société, dans sa bonté mal employée, ne lui assurerait pas le nécessaire dans un asile d'idiots ${ }^{58}$. La note relative au sacculus fournie par Nordau est par ailleurs intéressante, car il y explique sa comparaison zoologique :

Le sacculus est un cirripède qui vit à l'état de parasite dans le canal intestinal de certains crustacés. Il représente la plus profonde transformation régressive d'un être vivant primitivement d'une organisation plus élevée. Il a perdu tous ses organes différenciés et ne forme plus en substance qu'une vésicule (d'où son petit nom : petit sac) qui s'emplit des sucs de l'hôte absorbés par le parasite à l'aide de 
quelques vaisseaux qu'il envoie dans la paroi intestinale de celui-ci. Cet être rabougri a si peu gardé de choses d'un animal indépendant, qu'on l'a tenu longtemps pour une excroissance maladive de l'intestin de son hôte. ${ }^{59}$ l'homme dans la nature, ses limites et son avenir. La notion de Buffon appliquée à la zoologie n'est souvent présente dans la littérature que de façon anecdotique. Elle n'apparaît que lorsqu'il est mention de questions agricoles, notamment d'élevage, pour expliquer les différences de race de chevaux, de chiens, etc. Jusqu'aux années 1850, la dégénération discute finalement des possibilités évolutives du corps humain. Elle est ainsi toujours examinée conjointement au concept de perfectibilité. Elle revêt aussi dès la fin du xviiie siècle, une idée morale, poussée par la volonté de se conformer aux écrits bibliques et surtout de les justifier. La civilisation, malade, est assimilée à une sorte de corps qui dégénère. Croire au perfectionnement du corps, c'est s'affirmer transformiste. Le corps de l'homme peut ainsi soit régresser pour descendre dans l'échelle humaine, soit se perfectionner pour atteindre un statut normalement réservé aux êtres élus dans les textes religieux. Les fantasmes de la perfectibilité ont déjà provoqué, dès la fin du XVIII siècle, une libération du corps humain : l'homme peut désormais voler ou nager par le biais de machines. La technologie, vient, dès cette époque, compenser la lenteur de l'évolution biologique. Quant à la dégénérescence, elle alimente et justifie les idéologies pré-eugénistes et eugénistes de la fin $d u x x^{e}$ siècle et $d u x^{e}$ siècle, et pour ses compromissions avec la morale, elle dépasse l'étude du corps humain et elle a des répercussions, entre autres, dans le champ artistique.

\section{NOTES}

1. La notion de perfectibilité n'est cependant pas propre au XIX ${ }^{\mathrm{e}}$ siècle. La notion est définie et discutée pour la première fois par Jean-Jacques Rousseau dans Discours sur l'origine et les 
fondements de l'inégalité parmi les hommes en 1755. Cf. Florence Lotterie, «Les Lumières contre le progrès ? La naissance de l'idée de perfectibilité », in La recherche aujourd'hui, Dix-huitième Siècle, $\mathrm{n}$ - 30, 1998, Paris, PUF, p. 383-396.

2. Nous excluons de notre étude les textes anglais, car la notion, très influente en Angleterre, revêt parfois d'autres enjeux et soulève d'autres questions.

3. François-René de Chateaubriand, Génie du christianisme, Paris, Migneret, 1802, 4 vol.

4. Georges-Louis Leclerc, Comte de Buffon, Histoire naturelle, générale et particulière avec la description du cabinet du roi, Paris, imp. Royale, 1766, t. XIV, p. 311.

5. Claude Blanckaert, «Les fossiles imaginaires. Temps de la nature et progrès organique (1800 1850) ", Romantisme, $\mathrm{n}^{\circ}$ 104, 1999, vol.29, p. 87, [en ligne], http://www.persee.fr/doc/ roman_0048-8593_1999_num104_3410, [consulté le 5 octobre 2016].

6. Georges-Louis Leclerc, Comte de Buffon, Histoire naturelle, générale et particulière, supplément, Paris, imp. Royale, t. V, 1778, p. 27.

7. Johann Blumenbach, De generis humani varietate nativa, Göttingen, Vandenhoek et Ruprecht, 1775, $398 \mathrm{p}$.

8. Johann Blumenbach, De l'Unité du genre humain et de ses variétés, Paris, Allut, 1804, $313 \mathrm{p}$.

9. Jean-Claude de La Métherie (1743-1717), médecin, minéralogiste et géologue, est l'un des matérialistes les plus affirmés du XVIII ${ }^{\mathrm{e}}$ siècle. Cf. Henri de Blainville, « Notice historique sur la vie et les écrits de J.-C. Delamétherie ", Journal de Physique, de chimie, d'histoire naturelle et des arts, Paris, Cuchet, juillet 1907, t. 85, p. 78-107.

10. Jean-Claude de La Métherie, Considérations sur les êtres organisés: de la perfectibilité et de la dégénérescence des êtres organisés, Paris, Courcier, 1806, 462 p.

11. On peut le voir par le biais de l'outil Ngram viewer (https://books.google.com/ngrams), en demandant une visualisation de la fréquence d'utilisation des mots dégénération et dégénérescence dans les ouvrages $d u \mathrm{XIX}^{\mathrm{e}}$ siècle. Cette opération n'est réalisée que dans les ouvrages disponibles sur Google Livres.

12. Claude Bénichou, "Dégénération, dégénérescence, degeneration », Patrick Tort (dir.), Dictionnaire du darwinisme et de l'évolution, Paris, PUF, 1996, t. I, p. 1151-1157.

13. Julien-Joseph Virey, Nouveau dictionnaire d'histoire naturelle, appliquée aux arts, à l'agriculture, à l'économie rurale et domestique, à la médecine, etc., Paris, Deterville, 1817, p. 197.

14. Charles Dessalines d'Orbigny (dir.), Dictionnaire universel d'histoire naturelle, Paris, Renard, Martinet et $\mathrm{c}^{\mathrm{ie}}, 1841-1849$.

15. Frédéric Gérard, « Dégénérescence », Charles, Dessalines d'Orbigny (dir.), Dictionnaire universel d'histoire naturelle, Paris, Au bureau principal des éditeurs, 1844, p. 647.

16. Ibid.

17. François-René de Chateaubriand, « Génie du christianisme », Euvres complètes, Paris, Garnier frères, 1828 (1802), t. I, p. 64.

18. Ibid., p. 65.

19. Cf. Antoine Magnin, Charles Nodier naturaliste: ses cuvres d'histoire naturelle publiées et inédites, Paris, Hermann, 1911, 347 p.

20. Charles Nodier, "La Fée aux miettes ", Euvres complètes de Charles Nodier, romans, contes et nouvelles, Paris, Renduel, 1832, p. 215.

21. François-René de Chateaubriand, "Génie du christianisme ", Euvres complètes, Bruxelles, Tencé frères, t. 4, p. 167.

22. Ibid.

23. Louis de Bonald (1754-1840). Cf. Julien Damon, «La pensée de Louis de Bonald (1754-1840)», Informations sociales, $\mathrm{n}^{\circ} 122,2 / 2005$, p. 25 : www.cairn.info/revue-informations-sociales-2005-2page-25.htm, (consulté le 7 octobre 2016).

24. Louis de Bonald, «Du perfectionnement de l'homme », Euvres complètes de Louis Bonald, Paris, J.-P. Migne, vol. 2, p. 200. 
25. Nadja Durbach, «Londres, capitale des exhibitions exotiques de 1830-1860», in Pascal Blanchard, Nicolas Bancel, Gilles Boëtsch, Éric Deroo, Sandrine Lemaire \& Charles Forsdick, Zoos humains et exhibitions coloniales, 150 ans d'inventions de l'Autre, Paris, La découverte, 2011 (2002), p. 129.

26. Ernest-Théodore Hamy, "Quelques observations ethnologiques au sujet de deux microcéphales américains désignés sous le nom d'Aztèques ", Bulletins de la Société d'anthropologie de Paris, $\mathrm{n}^{\circ} 1,1875$, vol. 10, p. 39-72.

27. Nicolas Menut, "Les derniers Aztèques: Maximo et Bartola", in Pascal Blanchard, Gilles Boëtsch \& Nanette Jacomijn (dir.), Exhibitions, l'invention du sauvage, Arles, Actes Sud \& Paris, Musée du Quai Branly, p. 118. Ils apparaissent encore à Rouen en 1905 à l'affiche d'un spectacle du théâtre Bénévol. Journal de Rouen, me. 25 octobre 1905, n 208, p. 3.

28. Bénédict-Henry Révoil (1816-1882) est un romancier, journaliste et traducteur français. Cf. Notice de personne de la Bibliothèque Nationale de France, [en ligne], http://catalogue.bnf.fr/ ark:/12148/cb11921662q, (consulté le 10 octobre 2016).

29. Bénédict-Henry Révoil, Les Aztecs, Paris, Barba, 1855, 48 p.

30. Arthur de Gobineau, Essai sur l'inégalité des races humaines, Paris, Firmin Didot, 1853, t. 1, p. 39.

31. Charles Nodier, «De la Palingénésie humaine et de la résurrection », Euvres de Charles Nodier, Rêveries, Paris, Renduel, t. 5, p. 340.

32. Édouard Claparède, "Sur l'origine des espèces ", Revue germanique, octobre 1861, t. 17, p. 232-263.

33. Guy de Maupassant, "Le Docteur Héraclius Gloss ", La Revue de Paris, Paris, Bureaux de la Revue de Paris, novembre-décembre 1921, t. 6, p. 240.

34. Victor Hugo, Les Misérables, Paris, Émile Testard, 1890, « exergue ».

35. Bénédict-Auguste Morel, Traité des dégénérescences physiques, intellectuelles et morales et des causes qui produisent ces variétés maladives, Paris, Baillière, 1857, $700 \mathrm{p}$.

36. Ibid., p. 372.

37. Ibid.

38. Louis Bouilhet, «Les Fossiles », Euvres de Louis Bouilhet, Paris, Alphonse Lemerre, 1880, p. 145.

39. Ibid., p. 126.

40. Louis Boussenard, 10000 ans dans un bloc de glace, Paris, Flammarion, p. 23.

41. Ibid., p. 64.

42. Le premier conte paraît sous le titre de: «Hurlubleu, grand manifafa d'Hurlubière ou la perfectibilité. Histoire progressive », La Revue de Paris, t. 53, p. 155-180.

43. Bénédict-Auguste Morel, op. cit., p. 3.

44. Ibid., p. 5.

45. Charles Féré, La famille névropathique: théorie tératologique de l'hérédité et de la prédisposition morbides et de la dégénérescence, Paris, F. Alcan, 1898 (1894), p. 317.

46. Émile Zola, L'Assommoir, Paris, G. Charpentier, 1877, p. 365.

47. Ibid., p. 368.

48. Gustave Flaubert, Bouvard et Pécuchet, « Garnier-Flammarion », Paris, Flammarion, 1999 (mise à jour 2011), p. 376.

49. Ibid., p. 393.

50. Cf. Gilbert Darbouz, Dégénérescence et régénérescence dans l'œuvre d'Émile Zola et celle de Manuel Zeno Gandia, Berne, Peter Lang, 1997 et Louis Huard, «Le thème de la dégénérescence dans les Rougon-Marcquart d'Émile Zola », Thèse de la faculté de médecine, Cochin, 1988.

51. Émile Zola, Au Bonheur des dames, Paris, G. Charpentier et E. Fasquelle, 1883, p. 396.

52. Émile Zola, Germinal, Paris, G. Charpentier, 1885, p. 48.

53. Émile Zola, Le Docteur Pascal, Paris, Bibliothèque Charpentier, 1893, 390 p.

54. Alexandre Wenger, «Poésie et médecine au XIX ${ }^{\mathrm{e}}$ siècle. Les traductions françaises de Syphilis (1530) de Frascator ", in Muriel Louâpre, Hugues Marchal et Michel Pierssens (éd.), La poésie 
scientifique de la gloire au déclin, mis en ligne en janvier 2014, epistemocritique.org, (consulté le 10 octobre 2016).

55. Auguste Barthélemy, Syphilis : poème en deux chants, Paris, Béchet jeune et Labé, 1840, p. 38.

56. Nordau qualifie Darwin et Spencer «d'esprits médiocres»: Max Nordau, Dégénérescence, Paris, F. Alcan, 1894, t. 2, p. 339.

57. Paul Bourget, Essai de psychologie contemporaine, Paris, A. Lemerre, p. 24, in Max Nordau, op. cit ., t. 2, p. 106.

58. Max Nordau, op. cit., t. 2, p. 120.

59. Ibid.

60. Max Nordau, Dégénérescence, Paris, F. Alcan, 1894, t. 1, p. V et VI.

61. Jean-François Poirier, «Dégénéré Art», Encyclopaedia Universalis, [en ligne], http:// www.universalis.fr/encyclopedie/art-degenere/, (consulté le 7 octobre 2016).

62. Cf. également Lucia Rodler, "L'homme criminel de Cesare Lombroso: entre science et littérature ", Criminocorpus [en ligne], Histoire de la criminologie, 4. L'anthropologie criminelle en Europe, mis en ligne le 24 mai 2012, https://criminocorpus.revues.org/1893, (consulté le 6 octobre 2016).

63. Max Nordau, op. cit., t. 1, p. VII.

64. Ibid.

\section{RÉSUMÉS}

En 1766, Buffon tente de rendre compte des variations dans l'espèce humaine par un phénomène qu'il nomme dégénération. Repris par les naturalistes, étendu à l'ensemble du vivant, puis introduit dans la littérature, le concept séduit par sa puissance et par ses potentialités imaginaires. De Nodier aux romans d'anticipation de la fin du XIX ${ }^{e}$ siècle, le corps humain se dégrade pour rejoindre les espèces considérées comme inférieures ou au contraire se perfectionne pour se libérer des lois de la physique terrestre. Avec la réutilisation et l'élargissement de la notion par le médecin Morel dans son Traité des dégénérescences physiques, intellectuelles et morales et des causes qui produisent ces variétés maladives, paru en 1857, le terme devient une notion importante de la psychiatrie et se charge d'une connotation morale. De l'espèce à l'individu, du physique au psychique, le terme renvoie à de multiples réflexions dont nous souhaitons évaluer les enjeux.

\section{INDEX}

Mots-clés : dégénération, dégénérescence, perfectibilité, décadence, hérédité morbide, déclin

\section{AUTEUR}

\section{BÉNÉDICTE PERCHERON}

ANR/DFG BIOLOGRAPHES - Fondation Maison des sciences de l'homme de Paris, GRHis EA3831 